\title{
BSF Islands For Reduced Recombination In IBC Cells
}

\author{
Agnes A. Mewe, Nicolas Guillevin, Ilkay Cesar and Antonius R. Burgers \\ ECN Solar Energy, Petten, NL-1755 LE, The Netherlands
}

\begin{abstract}
We present a back junction back contact cell with small isolated BSF "island" areas, surrounded by emitter area. This is an alternative for the more common IBC cell with interdigitated fingers. The optimal cell lay-out was determined by device simulations. We used discontinuous emitter and BSF contacts, which we connected through floating metal fingers. Additionally, we applied a lighter front floating emitter diffusion. The performance increase predicted by the simulations was experimentally confirmed. The investigated design leads to a strong recombination reduction in our industrial IBC Mercury cell, leading to a $0.6 \%$ absolute gain in efficiency.
\end{abstract}

Index Terms - device simulation, interdigitated back contact, photovoltaic cells, recombination, silicon.

\section{INTRODUCTION}

The IBC Mercury cell is ECN's 6 inch industrial Interdigitated Back Contact cell design with a front floating emitter (FFE) that mitigates electrical shading. Although efficiencies above $21 \%$ have been reached [1], the performance is currently limited by the open circuit voltage, due to high recombination. The recombination activity in the cell is dominated by the emitter contacts and the heavily doped BSF area. Therefore, reducing both the BSF area and the emitter contact fraction is a route to decrease the recombination in the cell and therefore enhance the cell performance. This was explored both by simulations and experimental work.

Depending on the contact width and the screen printing tolerances, a minimum width of the passivated BSF area is required, which is typically more than $300 \mu \mathrm{m}$. In a onedimensional interdigitated finger design, the only option to reduce the BSF area fraction further is then to increase the emitter width, but this induces large transport losses. Therefore, we reduced the BSF length within the unit cell, and in this way we created "islands" of BSF surrounded by the rear side emitter.

Similar point-contact structures for the diffused areas have been studied before for IBC cells [2]-[3], but in these cases, the BSF islands were mainly created to study the electrical shading reduction benefits. In our case, electrical shading is not a major issue due to the collecting and transporting front floating emitter. Therefore, the BSF area reduction will mainly improve the passivation of the cell.

\section{SimulationS}

We simulated the cell lay-out in Quokka by taking the smallest possible representative part of the cell, which involves the half-widths of both the BSF and the emitter for an IBC cell. This unit cell is indicated in the IBC cell crosssection in Fig. 1 by the dashed line.

The Quokka device simulations include the influence of the metal contact area, but not of the metal grid resistance. Also cell features beyond the unit cell, such as busbars and cell edges, are not taken into account.

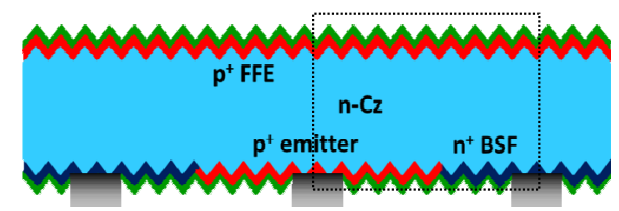

Fig. 1. Schematic cross-section of the IBC Mercury cell, with the standard unit cell indicated.

For the BSF island simulations, besides the width of the unit cell, also the unit length becomes a parameter, with the BSF length separately defined, as is shown in Fig. 2. This change leads to a 3D unit cell simulation.
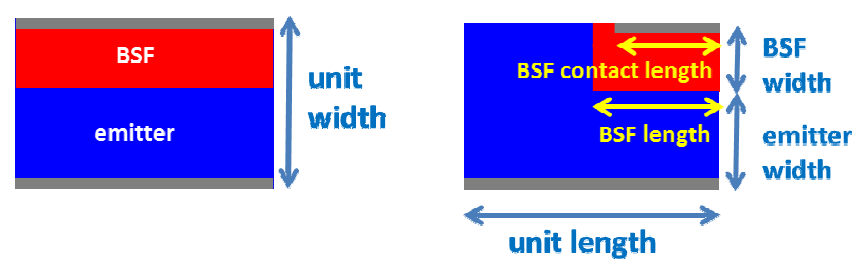

Fig. 2. Schematic view of the simulated rear side of the IBC Mercury cell, with the standard IBC unit cell (left) and BSF island lay-out (right).

\section{A. Unit Cell Studies}

In case of isolated BSF islands, the unit cell size determines the average travel distance for electrons to the BSF (i.e. transport losses), especially if the BSF fraction becomes very small. Additionally, a too small BSF contact area eventually becomes a limiting factor for effective transport.

To avoid these performance limitations, we decreased the unit cell size, which solved the BSF related transport limitations. The emitter contact length was kept equal to the unit cell length because of undesired current crowding effects. This caused the emitter contact to become very narrow, as the emitter contact faction was kept constant. However, in practice a certain minimum width is necessary due to screen-printing limitations. The solution to this will be discussed in the next section. 
IEEE PVSEC-44, Washington DC, June 25-30 2017

The lay-out of the optimized unit cell with BSF islands is sketched in Fig. 5 (left). The BSF area is reduced to $12.2 \%$ and the contact fractions of both emitter and BSF to $4 \%$ each. We calculate that a gain of $0.6 \%$ absolute in efficiency can be achieved compared to the reference case with $37 \%$ BSF fraction and $6 \%$ contact fractions.

\section{B. Sensitivity Studies}

As we use screen-printing for patterning the doped areas and for the metallization, there are two parameters that will become critical for a BSF islands design. Firstly, the size of the passivated BSF area needs to be large enough for good alignment of the BSF contact. Secondly, the emitter contact needs to have a certain minimum width because of metal paste printability. For both aspects, sensitivity studies were carried out to translate the optimal unit cell design from the simulations into a real unit cell that can be manufactured by screen printing.

The passivated BSF area fraction was varied between $8 \%$ and $60 \%$ and different shapes were used, to investigate how sensitive the area lay-out is to the performance. It appeared that the efficiency change is less than $0.05 \%$ absolute between 8 and 20\% BSF area fraction, as shown in Fig. 3. The performance is mainly a trade-off between $V_{o c}$ and $F F$. We concluded that we can use a BSF fraction of $20 \%$ as a safer option for metal contact alignment.

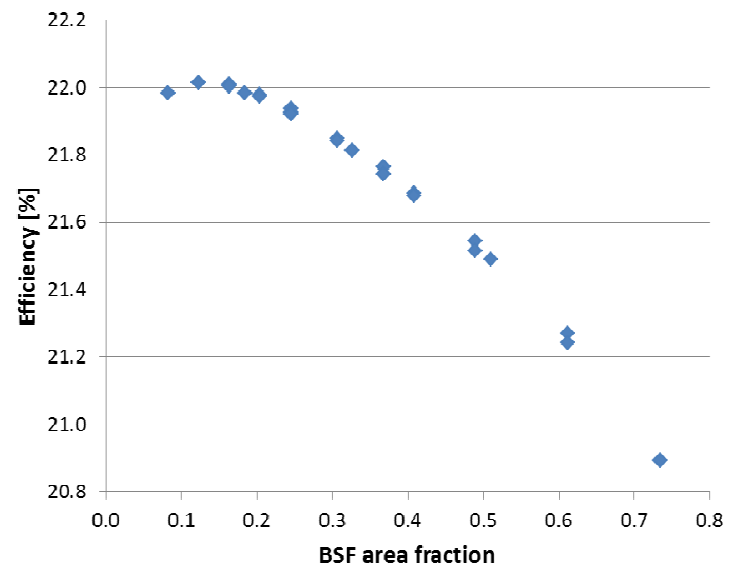

Fig. 3. Simulated cell efficiency as a function of BSF area fraction on the rear side of the IBC cell. A broad optimum between 8 and $20 \%$ BSF area fraction can be used.

The optimal emitter contact resulting from the simulations was quite narrow (less than $30 \mu \mathrm{m}$ wide) due to the small unit cell width and small metal fraction. To make the contact design compatible with screen printing, a sensitivity study to wider and shorter emitter contact fingers was carried out, while keeping the contact coverage the same. The simulations showed that doubling the width and halving the length results in printable dimensions and with a minor efficiency loss of
$0.05 \%$ absolute, as seen in Fig. 4. Therefore, this emitter contact design change was adopted.

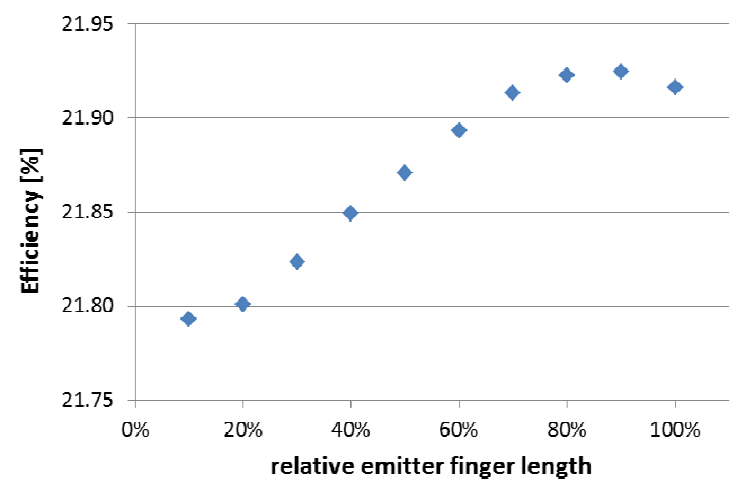

Fig. 4. Simulated cell efficiency as a function of emitter finger length relative to the unit cell length.

Besides the above modifications, we also changed the shape of the BSF area from square to circular. This is not expected to change the cell performance significantly, based on the sensitivity study of the BSF area size. The final two designs as used in the experiment are shown in Fig. 5 (right).

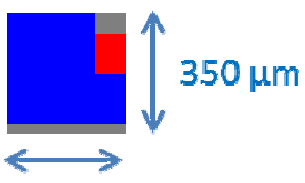

$350 \mu \mathrm{m}$

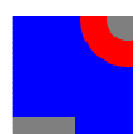

$\Uparrow 140 \mu \mathrm{m}$ $(12.5 \%$ BSF $)$

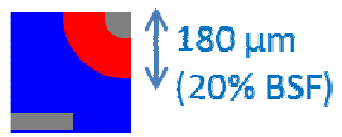

Fig. 5. Schematic view of the simulated 2D rear side of the IBC Mercury cell, after optimization of the unit cell size (left) and the two designs that followed from the sensitivity studies (right).

\section{EXPERIMENTAL}

The simulation results were used as input for the cell lay-out in the experiment. The experimental procedure and results are presented in the next sections.

\section{A. Experimental Procedure}

The two unit cell designs with different BSF area fractions, derived from the optimal simulation design and sensitivity studies, were applied to a full-size 6 inch IBC solar cell layout. As the metal contacts of both the emitter and BSF are interrupted, the contacts of each polarity are connected by screen printed metal fingers that do not etch through the $\mathrm{SiN}_{\mathrm{x}}$ passivation layer ("floating fingers").

As an additional parameter, we applied a light front floating emitter, which was combined with the smallest BSF islands, as this configuration is most sensitive to a further decrease of 
IEEE PVSEC-44, Washington DC, June 25-30 2017

recombination sources. These cells are expected to yield the highest performance by an extra $J_{s c}$ and $V_{o c}$ increase, based on previously published passivation results of lightly doped emitters [4].

\section{B. Experimental Results}

The analysis of the cell results revealed that the cells with BSF islands were all shunted. We observed unexpected busbar paste spreading near the edges of the cell, short-circuiting to the fingers of opposite polarity.

To mitigate the influence of the shunts in the analysis, the edges were removed by laser scribe-and-break $10 \mathrm{~mm}$ from each edge, indicated with the red dashed lines in Fig. 6. This edge removal leads to edge recombination losses, especially due to the minority carrier transport of the front floating emitter. For fair comparison of the different cells, also some of the cells with the reference process were laser-cut.

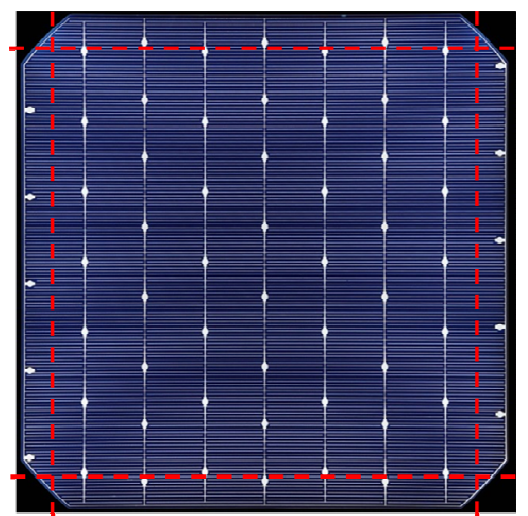

Fig. 6. Position of the laser scribes to remove the cell edges.

The edge removal causes a $3 \%$ relative efficiency loss (exclusively due to $J_{s c}$ loss, from 40.0 to $38.8 \mathrm{~mA} / \mathrm{cm}^{2}$ ) in the reference cells. In Fig. 7, only the results of the reference cells after removal of the edges are presented.

As shown in Fig. 7, $J_{s c}$ and $V_{o c}$ both increase for the cells with BSF islands with respect to the reference. The average $J_{s c}$ gain is 0.4 and $0.7 \mathrm{~mA} / \mathrm{cm}^{2}$ for medium and small islands, respectively, and the $V_{o c}$ gain is 4 and $6 \mathrm{mV}$. This is in qualitative agreement with the simulation results $\left(J_{s c}\right.$ gain of 0.4 and $0.5 \mathrm{~mA} / \mathrm{cm}^{2}$, and $V_{o c}$ gain of 7 and $9 \mathrm{mV}$ ).

The average $F F$ loss of the cells with medium islands compared to the reference $(0.4 \%$ absolute; not shown), is also well in line with the simulation results. For the cells with small BSF islands, we observe a higher $F F$ loss than expected from the modeling. After further analysis of the cells, we can attribute most of the $F F$ loss to pseudo-FF differences. The series resistance increase for the small BSF islands is relatively small. Most of cells with the small BSF islands also suffer from extremely low shunt resistance values, which results in even lower $F F$. Those cells are taken out of the analysis. The low shunt values are ascribed to misalignment between the small BSF islands and the BSF metal contact, although this assumption needs to be verified by characterization.

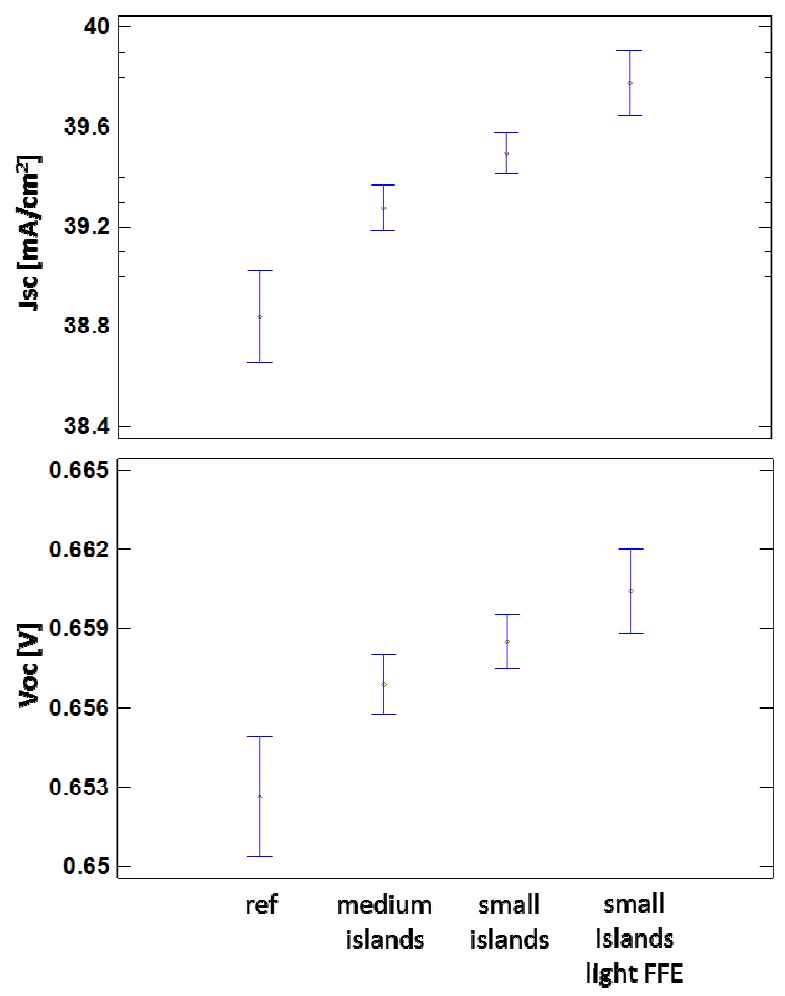

Fig. 7. $J_{s c}$ and $V_{o c}$ results of the cells after removal of the edges. Both $J_{s c}$ and $V_{o c}$ show a steady increase towards smaller BSF area fraction and lighter front side doping.

To complete the cell comparison, we compare the best cells of each group, as listed in Table 1. Although the reference cells show a lower performance level after the edge removal, it is clear that the use of small BSF areas and a small unit cell is beneficial, especially when it is combined with a lowly doped front floating emitter. The best cell of this type obtained a $0.6 \%$ absolute higher efficiency than the best reference cell.

TABLE I

Summary of Best Cell Results After Edge Removal

\begin{tabular}{|l|c|c|c|c|}
\hline & $\begin{array}{c}\mathrm{J}_{\mathrm{sc}} \\
{\left[\mathrm{mA} / \mathrm{cm}^{2}\right]}\end{array}$ & $\begin{array}{c}\mathrm{V}_{\mathrm{oc}} \\
{[\mathrm{V}]}\end{array}$ & $\begin{array}{c}\mathrm{FF} \\
{[\%]}\end{array}$ & $\begin{array}{c}\text { Efficiency } \\
{[\%]}\end{array}$ \\
\hline Reference & 38.9 & 0.653 & 79.1 & 20.1 \\
\hline Medium islands & 39.4 & 0.656 & 78.8 & 20.4 \\
\hline Small islands & 39.7 & 0.657 & 77.7 & 20.3 \\
\hline $\begin{array}{l}\text { Small islands } \\
\text { and light FFE }\end{array}$ & 39.9 & 0.663 & 77.9 & 20.6 \\
\hline
\end{tabular}




\section{DISCUSSION}

\section{A. Device Simulations}

The device simulations were of great assistance to define the optimal design parameters and sensitivity of the IBC cell. All resistance and recombination contributions could be separately listed in the simulation output, which was very helpful in identifying the limiting factors that needed further optimization.

The reduction of the highly recombinative BSF area fraction and of the metal contact fractions led to an increase in $J_{s c}$ and $V_{o c}$. The observed trend in $J_{s c}$ was not completely predicted by the simulations, so this needs further attention.

At the same time, a reduction of the unit cell size was necessary to keep the (majority) transport losses low. This is confirmed by the $F F$ trend for medium islands, that follow the simulation and is almost as high as the $F F$ of the reference.

\section{B. Edge removal of cells}

Some uncertainties arise in considering the impact of the edge removal to the cells with $\mathrm{BSF}$ islands compared to reference IBC cells. Although the properties of the cell edges are mostly similar for reference cells and BSF island cells, we cannot be sure that the impact is exactly the same, and therefore, we cannot be sure that the observed performance gain will be the same for full-size cells. For that reason, a final answer to this question can only be given for cells without edge cuts in a second experiment.

\section{CONCLUSIONS}

We presented an IBC cell with adjusted doping and contact pattern on the rear side, using small isolated BSF areas (BSF islands) surrounded by emitter, accompanied by a decrease of ton DC, June 25-30 2017

the unit cell size. Full-size 6 inch IBC cells were processed, and the cell edges were removed because of the shunts at these locations.

The BSF island design boosts the $J_{s c}$ and $V_{o c}$ of the cell, especially if it is combined with a lightly doped front floating emitter, resulting in $0.6 \%$ absolute increase in efficiency for the best cell, compared to the best reference cell.

The removal of the edges causes the lower efficiency level of the cell results. A repeat of the experiment on full-size cells will be performed to provide final conclusions on the performance of the BSF island design.

\section{ACKNOWLEDGEMENTS}

This work was supported by the Dutch Ministry of Economic Affairs within the TKI project IBCense.

\section{REFERENCES}

[1] P. Spinelli, P. Danzl, N. Guillevin, A. A Mewe, S. Sawallich, A. H. G. Vlooswijk, B. W. H. van de Loo, W. M. M. Kessels, M. Nagel and I. Cesar, "High-resolution sheet resistance mapping to unveil edge effects in industrial IBC solar cells", in Energy Procedia 92, 2016, p. 218.

[2] C. Reichel, F. Granek, M. Hermle and S. W. Glunz, "Backcontacted silicon solar cells with insulating thin films", at $n p v$ workshop, 2011

[3] J. Haschke, N. Mingirulli, and B. Rech, "Progress in point contacted rear silicon heterojunction solar cells", in Energy Procedia 27, 2012, p. 116

[4] A. A. Mewe, P. Spinelli, A. R. Burgers, A. H. G. Vlooswijk, N. Guillevin, E. J. Kossen and I. Cesar, "Emitter and contact optimization for high-efficiency IBC Mercury cells", in Proc. $32^{\text {nd }}$ EUPVSEC, 2016, p. 760 